\title{
A Random Access Scan Architecture to Reduce Hardware Overhead
}

\author{
Anand S. Mudlapur, Vishwani D. Agrawal and Adit D. Singh \\ Auburn University \\ Dept. of Electrical and Computer Engineering \\ Auburn, AL 36849 \\ anand,agrawvd,singhad@auburn.edu
}

\begin{abstract}
The concept of Random Access Scan (RAS) where every Flip-Flop is addressed uniquely has been subject to criticism at the very thought. It seems at the first impulse that the cost associated with routing is overwhelming. This argument has shelved the idea for 25 years now. In this paper we propose an architecture that minimizes the signals to the RAS Flip-Flop (FF) and give an estimate of the increase in area due to the increase in gates and increase in routing. Two global signals, scanin and mode control, have been eliminated from the previous $R A S$ designs presented in the literature. For $n$ flip-flops, instead of routing $n$ address wires, one to each FF, we use $\sqrt{n}$ wires in an xy matrix layout. A unique toggle mechanism is incorporated in the RAS FF that totally eliminates the scanin signal wire and reduces the vector set up to $60 \%$ compared to traditional serial scan (SS). The SS induces unnecessary circuit activity during scan and the circuit under test (CUT) dissipates an enormous amount of power. Our design reduces the power dissipation by 99\%. The problem of delay testing is highly constrained in SS and the scan-cell is often modified to assist delay testing. Any single input change delay test can be directly applied in our design. Hence all testable paths in the circuit can be effectly tested without constraints. We also propose a multistage scanout system to observe the addressed FF avoiding a slow output bus with very high capacitance.
\end{abstract}

\section{Introduction}

Testing sequential circuits has been one of the most challenging areas in digital circuits. Automating test generation for large sequential circuits without Design for Testability (DFT) logic has met with marginal success. Additional hardware is usually added to accom- plish a high fault coverage in these circuits. Serial scan (SS) design has been one of the most successful methods in testing digital circuits. Although it enables the application of combinational test generation algorithm, alternative methods are sought after because of some inherent drawbacks like increased test time and test power consumption. Several methods are suggested and implemented to circumvent this problem. A widely successful method is partial scan [1]. But it provides a trade off between the ease of testing and the costs associated with scan design. The problem of efficiently selecting the scan registers is still widely open to research. Cross-check methodology [2] provides a comprehensive solution to test sequential circuits and almost solves all the problems related to test application time and provides massive controllability and observability.

Power consumption during testing is much higher than during normal circuit operation. It is important and vital to target, low power dissipation during testing, since excessive heat can damage the circuit under test. The long scan-in/scan-out sequences trigger random circuit activity resulting in high power consumption. Test scheduling is a common approach to avoid the damage of complex devices, such as SOC [3, 4]. As a result test parallelism is reduced and testing time eventually increases. It is a well known fact that serial scan operation may create unacceptably high activity due to frequent transitions in scan chain. To circumvent this problem the scan clock is slowed down [5]. Again the test application time increases, which is undesirable.

ATPG based methods have also been used to target the power issue [6]. However, this method often results in longer test sequences. Compaction of test vectors, can reduce the length of tests, but the compacted vector set generally induces more activity resulting in higher power consumption [7]. To overcome this problem modification of test vectors for power saving has also been addressed [8]. Another method studied to 
reduce test power and/or test application time is modifying the order of scan cells or inserting inversion logic between scan cells after the test generation [9]. Seth et al. in [10] describe a double-tree scan architecture to reduce test power. Although the power saving is quite significant, the test time and test data volume either remains the same or increase. A modified scanarchitecture to reduce test time in full-scan circuits has been addressed [11]. The authors illustrate a reduction of test time by $50 \%$, nevertheless test power still remains a matter of concern.

Testing for path delay faults in non-scan sequential circuits is complicated by the limited state transitions during normal operation. An accepted method for overcoming this difficulty is to use a scan chain consisting of enhanced scan FFs which makes the application of arbitrary vector pairs possible. However this technique requires a hold-latch connected to each FF in addition to a "HOLD" signal that must be routed to every hold latch. This increases the area overhead and also adds some delay in the scan path [12]. Normalscan sequential circuits can be tested for delay faults, but the vector-pairs must be specially generated [13]. Here, the first vector V1 is scanned in (usually with a slow scan clock) and is then replaced in the scan register by either (a) applying V2 which is obtained by a one-bit shift to the scan register also known as scanshift delay test $[14,15]$, or (b) propagating V1 through the combinational logic in the normal mode, where the state portion of V2 my be justified by V1, known as Functional broad-side delay test [16]. However, high fault coverages are dependent on the circuit and cannot be guaranteed due to the correlation between the two vectors.

All the problems stated above are due to the underlying architecture used, which is SS! Random access scan (RAS) [17] is a single solution to most of them. As the name implies, each scan-cell is randomly and uniquely addressable. A recent RAS architecture [18] targets reduction of both test application time as well as power consumption simultaneously, which are otherwise complementary objectives. A modified scheme of RAS has been described in [19], although with a different name. Here, the captured response of the previous pattern in the FFs is used as a template and modified by a circular shift for the subsequent pattern.

In this paper, we describe an architecture for the RAS-cell, aiming at minimizing the routing complexity as compared to the architecture described in the previous work [18]. This work is based on the premise that as technology advances (more gates per die), a minimal increase in design for testability (DFT) at the cost of reduced computational resources and increased flexibility in testing, will be least prohibitive.

This paper is organized as follows. The architecture along with an optimized routing of decoder signals, and the testing of the test-circuitry is described in Section 2. An algorithm to compact the test vectors is described in Section 3. In Section 4, delay testing using RAS is investigated. Experimental results on ISCAS Benchmark circuits are presented in Section 5. An algorithm to generate test vectors especially for RAS technique is described in Section 6 .

\section{New RAS Architecture}

In $\mathrm{SS}, \mathrm{FFs}$ form a seamless chain from the scan-in pin to the scan-out out pin in the test mode, forming a shift register structure. During normal mode of operation the input to the FFs is from the combinational logic. During scan-in/scan-out, every FF is subject to change in state. This leads to continuous activity in the FFs as well as the combinational circuits dissipating a lot of power, which is very undesirable. In RAS, a decoder is used to address every FF. Hence at any given point of time only one FF is accessed while the other FFs retain their state. This way no activity takes place in the circuit during the scan mode or the test mode. The architectures described in the literature $[17,18,20]$ mainly consists of a scan-in signal that is broadcasted to all the FFs, a test control signal that is also broadcasted to all the FFs and a unique decoder signal from the decoder to every FF. The output from the FF is either fed into a MISR or the outputs are ORed to a primary output justifying the logic.

The design could become cumbersome if a unique decoder signal is routed to every FF and the scan-in signal is broadcasted. In the design that we have developed, we use a unique toggling scheme wherein the addressed FF toggles its present state in the test mode, there by reducing a separate globally routed scan-in signal. The output from the FF is fed into a bus. Thus the addressed FF places its value on the bus in the test mode providing the necessary observability.

The design of our RAS FF can be described by three operations that are essential to satisfy the test requirements, which are, to capture the response of the circuit in the normal mode, to toggle the current state of the FF being addressed and retrieve the contents simultaneously, and finally make sure that all unaddressed FFs hold their previous states while one FF is being accessed during test mode. The operations are summarized in the first column of Table 1 . We have assumed that the FF is made up of a master and a slave latch similar to that shown in Figure 1.

Every FF gets two inputs, one from the row (x) and 


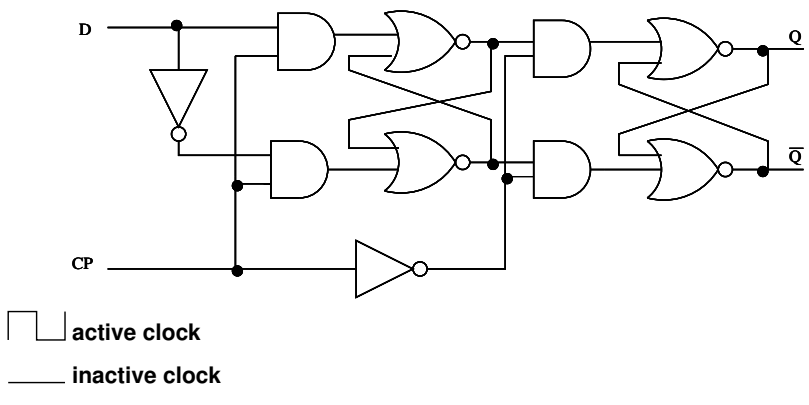

Figure 1. Master-Slave FF.

Table 1. RAS signals.

\begin{tabular}{|c|c|c|c|}
\hline \multirow[t]{2}{*}{ Function } & \multirow[t]{2}{*}{ Clock } & \multicolumn{2}{|c|}{ Address decoder outputs } \\
\hline & & Row (x) & Column (y) \\
\hline $\begin{array}{c}\text { Normal } \\
\text { data }\end{array}$ & active & 0 & 0 \\
\hline \multirow{2}{*}{$\begin{array}{c}\text { Toggle } \\
\text { data }\end{array}$} & $\overline{\text { inactive }}$ & $\overline{\overline{1}}$ & "active clock \\
\hline & inactive & active clock & 1 \\
\hline \multirow{3}{*}{$\begin{array}{r}\text { Hold } \\
\text { data }\end{array}$} & inactive & 1 & 0 \\
\hline & inactive & 0 & 1 \\
\hline & inactive & 0 & 0 \\
\hline
\end{tabular}

one from the column (y) decoder. The other inputs are clock and data from the combinational logic. The combinations used for the three defined functions are listed in Table 1. The operation of the modified scanFF can be described using Figure 3.

In the normal mode of operation, the $\mathrm{x}$ and $\mathrm{y}$ lines are ' 0 's and the decoders are disabled. The output at every AND gate inside the FF is ' 0 ' enabling the OR gate and routing the data from the combinational logic through the multiplexer to be captured in the FFs. The master is latched at the pulse of the clock and the slave is latched subsequently. In the test mode, the clock is stopped and the row and column decoders select one line each to address a FF at its intersection. Hence only one $\mathrm{FF}$ which is addressed sees a ' 1 ' at both $\mathrm{x}$

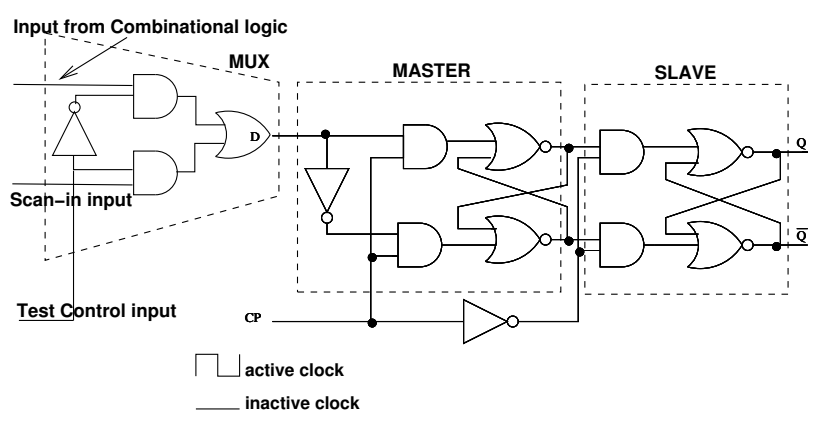

Figure 2. serial scan-FF.

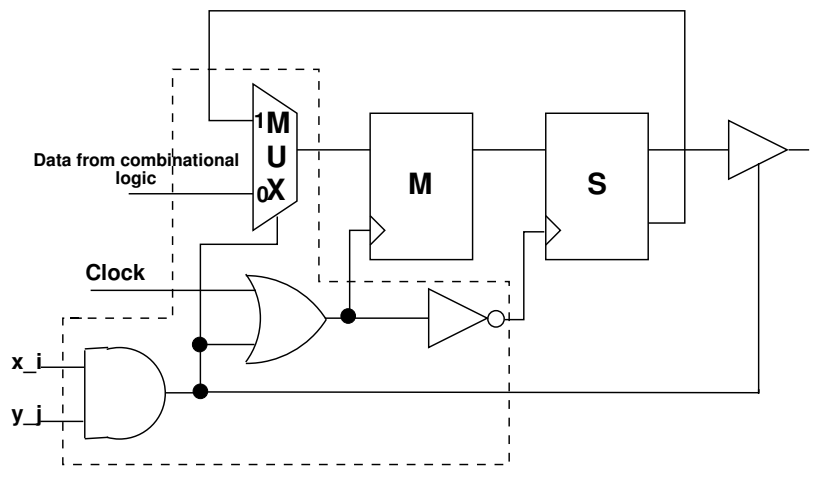

Figure 3. Modified scan-FF to implement RAS.

and y lines. The multiplexer now routes the inverted contents of the FF to the master, we address this as the toggle mode. The signal on the $\mathrm{x}$ or $\mathrm{y}$ is then made to go to ' 0 ', performing the function of a clock to load the slave latch. This operation can happen at any desired frequency. Hence the addressed FF toggles its state and at the same time the tristate buffer is enabled to route the data previously stored in the FF to a common bus. Meanwhile, the other FFs have to hold their previous states while the toggle operation is being performed on one FF. Since the output from the AND gate is ' 0 ', the master latch never gets activated since the clock is turned off and hence the slave latch holds its previous state. One must note that addressing a FF reads the contents of the FF as well as toggles its contents. Hence the contents of the FF after a read operation would be opposite to the value that was read out. Care is to be taken to avoid race condition in the FF. This can be achieved by inserting appropriate delays.

All the FFs can be cleared initially by using a builtin circuitry, which in the clear mode would read each $\mathrm{FF}$ and based on its current contents determine if another read operation is to performed to clear it. For example, during the clear mode, if a FF is read and is found to contain logic ' 0 ' state then the same FF is addressed again to toggle its present state which is logic ' 1 '. This calls for two clock cycles. While in the case when the first read is a logic ' 1 ', the next cycle is a dummy cycle and the FF is left unaddressed. Hence, the number of clock cycles to clear all FFs would be twice the number of FFs in the circuit.

The row and column decoders are built in such a way that the row and column lines intersect to address a FF. This design has the least area and routing overhead compared to other decoding schemes. The total number of rows and columns depends on the number of FFs and the actual layout. The least number of hor- 


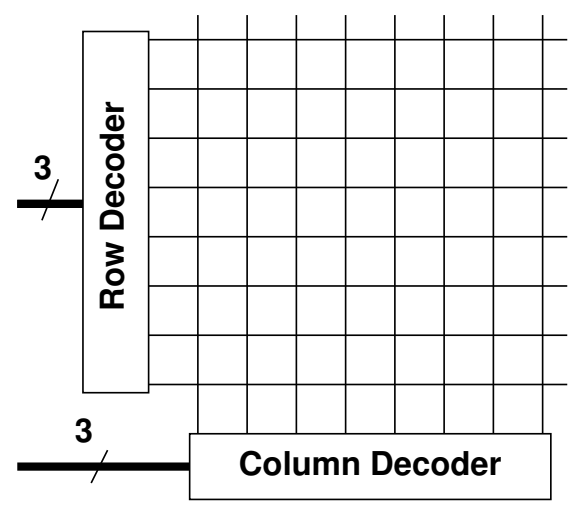

Figure 4. Decoder design.

izontal and vertical lines would occur in the case when both are equal in number and numerically equal to the square root of the number of FFs in the circuit. Let us assume that the row decoder decodes 1 among the $\mathrm{m}$ lines and the column decoder decodes 1 among the $\mathrm{n}$ lines, where the total number of FFs is $\mathrm{m} \times \mathrm{n}$. It is assumed that the inputs to the decoder fans-out from the primary inputs of the circuit. Therefore the number of inputs to the circuit must be greater than $\log _{2} \mathrm{~m}$ $+\log _{2} \mathrm{n}$.

In comparison with cross-check [2] where an entire row needs to be addressed and a single FF can be set only if the contents of all other FFs in that row are known. And this scheme wouldn't work if a MISR is used to capture the outputs. In our architecture we can address any FF without any constraint and read the value for it correspondingly.

\subsection{Routing}

The architecture described in [18] used three separate signals to control any given $\mathrm{FF}$ apart from the signal feeding-in from the combinational logic. This design is illustrated in Figure 5. Our design performs the equivalent function using only a decoder signal. There by eliminating two globally routed signals to the FF. The output from every FF is connected to a bus that leads to a primary output pin. This is analogous to the "Test-control" signal being routed in the SS, only that the TC signal is connected to every FF from a primary input pin. The scan-in signal, which forms a chain from a primary input to a primary output through all the FFs in SS is eliminated and a signal from the decoder to each FF is added. The conventional decoder scheme used in [18] becomes very complex and cumbersome to implement since a single wire would have to be routed to every FF. Also the decoder complexity will grow proportionally. For 65,536 (64K) FFs, 65,536 unique wires will have to be routed across the IC and would require 64K 16-input AND gates to decode 16 address lines. The output of the FFs are fed to a MISR, i.e., every FF feeds to an MISR in the previous RAS designs.

The grid architecture shown in Figure 4 was found to be the most efficient way to layout the decoders. The total number of extra routes added is $m+n$. With a minimum of two layers of metal routing, the row wires can be accommodated within the channel inbetween the cell rows and the column wires can be routed over the cell in the next metal layer. Hence there will be an increase of one track per channel (assuming m channels) and $n$ tracks that are routed on the next metal layer. Let us assume a circuit with 65,536 (64K) FFs like before. Let us also assume a square layout that has 256 routing channels. Hence every row will contain 256 FFs. i.e. $\mathrm{m}=256$ and $\mathrm{n}=256$. The total number of additional tracks will be $256+256=512$. Let the length of every channel be ' $\mathrm{l}$ ' $\mu \mathrm{m}$ and assuming the vertical dimension to be a linear multiple of the channel length, i.e., $(\mathrm{q} \times \mathrm{l}) \mu \mathrm{m}$, then the increase in length of routes is $(\mathrm{q}+1) \times 1 \mu \mathrm{m}$. Hence 65,536 wires have been reduced to 512 wires.

\subsection{Scanout Design}

We have designed a novel mechanism for the scanout of the FFs. It is a hierarchical structure that ensures there is no loading on the FFs while driving the output. The idea is illustrated in Figure 6. A cluster of FFs in a close proximity, feed to a common bus. And the contents of the bus are captured by a normal FF clocked by the normal clock which is suppressed in the test mode to the rest of the circuit. The row address that activates the FF is also captured in another FF. The contents of the FF are propagated further in the next clock cycle. This scheme was developed considering the drivability of the tri-state buffers. The outputs are pipelined to minimize the delays that may have resulted without the hierarchical structure. It is intuitive from Figure 6 that the succeeding stage would have the outputs enabling the tristate buffers, ORed and fed to a FF to preserve the address. We are also evaluating a scheme with sense amplifiers and pre-charged lines read the contents of the FFs.

\subsubsection{Area Overhead}

Assuming a circuit has $n_{g}$ gates and $n_{f f}$ FFs each consisting of 10 gates. Assuming the FF is designed as shown in Figure 1, the gate overhead of SS [12] and RAS is given by equations (1) and (2), respectively 


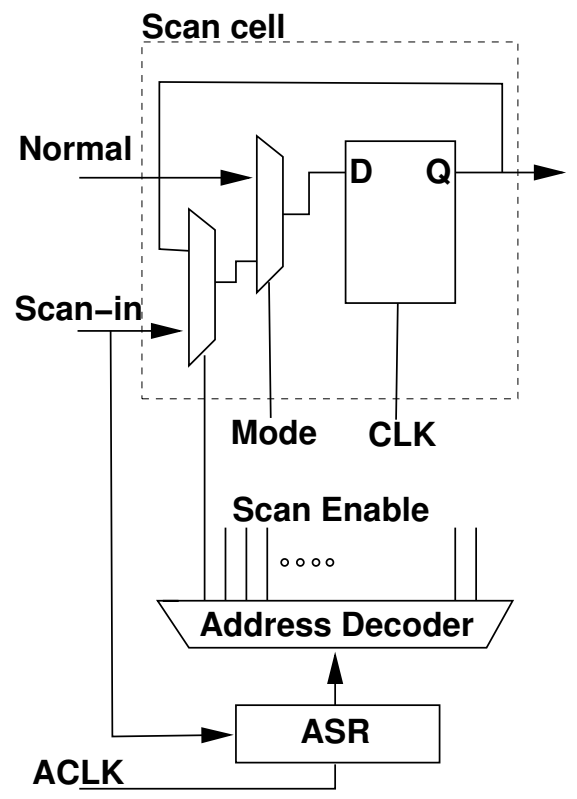

Figure 5. Design of RAS as described in [18].

Gate overhead of scan $=\frac{4 \times n_{f f}}{n_{g}+10 \times n_{f f}} \times 100 \%$

The RAS FF has 4 gates of the multiplexer similar to scan-FF and the gates in Figure 1, the additional gates that are added are one AND-OR-INVERT (AOI) and a tri-state buffer as shown in Figure 3, i.e., the logic can be minimized by using one complex gate (AOI) and using the same inverter that is used to invert the clock in a FF. The logic shown within the dotted box in Figure 4 can be further minimized. For the number of gates increased by the decoder, let us assume a decoder structure built using pass transistors. The number of transistors required to decode ' $\log _{2} \mathrm{c}$ ' lines to 'c' lines approximately equals $2 \times$ c. Assuming that a gate is made up of 4 transistors and $\mathrm{n}_{f f}=\mathrm{c}$ (horizontal lines) $\times d$ (vertical lines), the gate overhead of RAS can be approximated by the equation shown below:

$$
\text { Gate overhead of } R A S=\frac{6 \times n_{f f}+\frac{c+d}{2}}{n_{g}+10 \times n_{f f}} \times 100 \%
$$

Let us consider a circuit with 5,120 gates and assume that there are 512 FFs in the circuit. The gate overhead of serial scan is $20 \%$ from Equation 1 and the gate overhead of RAS is $30.2 \%$ from Equation 2. Hence there is an increase of $10 \%$ in the $\mathrm{x}$ dimention of the layout.

\subsection{Testing}

The tests target all the stuck-faults in the CUT. Consistently dominant faults are modeled on the tristate buffers in the circuit $[21,22]$. The decoder is first tested using the MATS $++[23]$ test. The FFs are cleared initially since it is assumed that a clear operation is possible on all the FFs to initialize them and then the test is performed.

$$
\{\Uparrow(\mathrm{w} 0) ; \Uparrow(\mathrm{r} 0, \mathrm{w} 1) ; \quad \Downarrow(\mathrm{r} 1, \mathrm{w} 0, \mathrm{r} 0)\}
$$

This test adequately tests for Address decoder faults (AF) unlinked with transition faults (TF) and all AFs linked with TFs. All the stuck at faults (SAF) are detected because, from each cell a ' 0 ' and a ' 1 ' is read uniquely.

\section{$\mathbb{\Downarrow}$ - Addressing order can be either increasing or decreasing}

介- Increasing memory addressing order

$\Downarrow$-Decreasing memory addressing order

After the test-circuitry is tested for fault free operation, the FFs are set up to perform the routine tests. The initial states are loaded into the FFs and the combinational inputs are applied at the primary input. The vector sequences required to test the decoder and FFs, is linearly proportional to the number of FFs in the circuit.

\section{Algorithm to Compact Test Vectors}

A greedy algorithm is developed to compact the test vectors. Here the vectors for the combinational circuit are obtained using an $\mathrm{ATPG}^{1}$. The vectors are sequenced based on the response captured by the FFs for an input vector along with the change in state of those FFs that are read where the faults have propagated during the application of the previous vector. The algorithm is as follows:

1. Obtain the combinational vectors along with good circuit responses and store the results in a stack

2. Find the FFs where faults are propagated at each vector

3. While number of vectors $>0$

(a) Read all the FF where the faults are detected

\footnotetext{
${ }^{1}$ Vectors were obtained from HITEC/PROOFS [24] and circuit responses and outputs where faults were detected on each vector were obtained using AUSIM [25]
} 


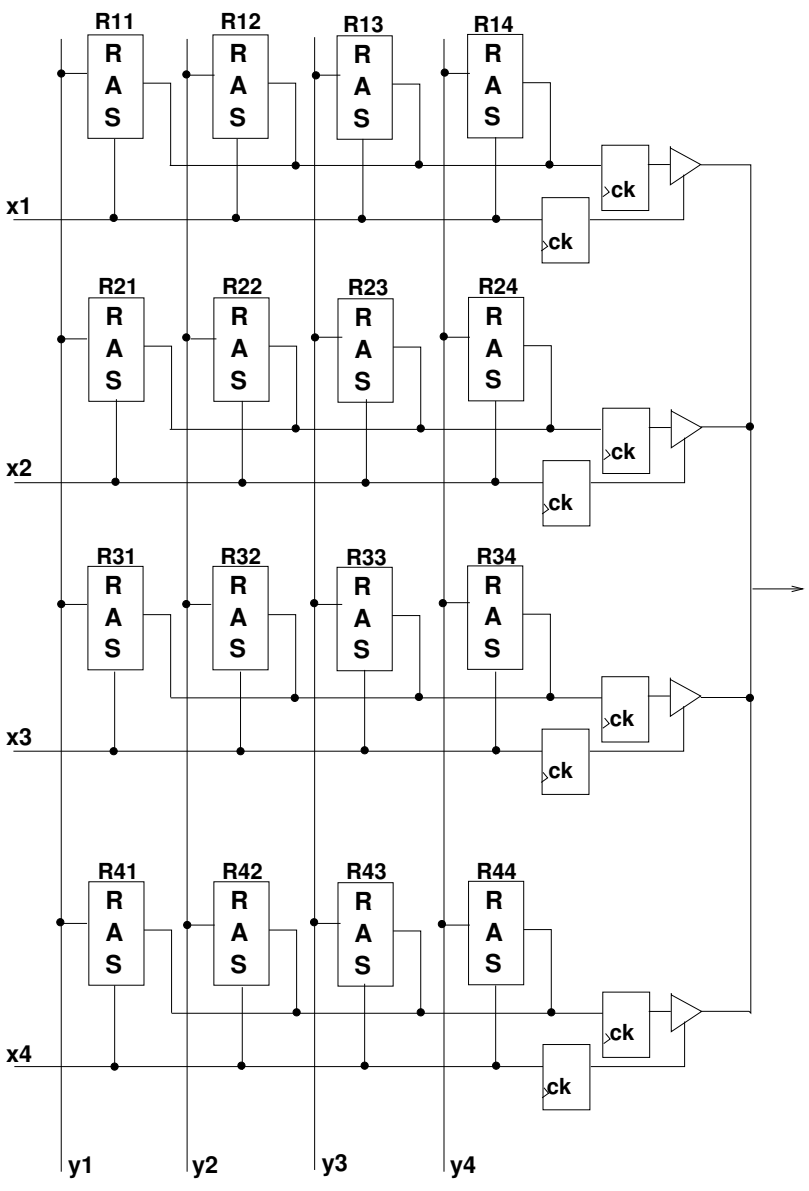

Figure 6. Routing of decoder signals in RAS.

(b) Choose the next vector from stack that has least hamming distance from current FF states

\section{End While}

During scan-in, the CUT is subject to unnecessary activity and all the FFs are subject to change state. Various methods are presented in the literature to mask the FF transitions during test mode [26, 27]. Assuming that the power dissipation in the CUT, is directly proportional to the number of transitions in the primary inputs and the transitions in the states of FFs, the power dissipation in RAS is reduced drastically, since, the only activity during scan mode is the transition in state of a single FF under consideration and transitions at the primary input pins that control the decoder.

\section{Delay Testing Using RAS}

Delay testing in SS circuits is very constrained. The scan-FFs are modified and HOLD latches [28] are of- ten inserted between the FFs and the combinational logic. The latches insert excess delays in the path and increase area overhead due to routing of an additional control (HOLD) signal. A one bit change can be obtained very easily using RAS, which is very vital in the case of delay testing. A vector $\mathrm{V} 1$ is set up and vector V2 with a one bit change is applied. It is known that any testable path can be tested by a single input change vector pair [29]. These tests are easy to apply in RAS but cannot be guaranteed in SS. A change in state of a FF, only needs one clock and the circuit response is captured in the next clock cycle, there by testing a desired path for delay. Hence delay testing, can be performed using RAS with no additional hardware, and any combinationally generated delay test vector will work for sequential circuits using RAS.

Error diagnosis, which is a lengthy process for SS, can be very efficient with RAS.

\section{$5 \quad$ Results}

The proposed architecture was modeled and tested on ISCAS benchmark circuits. The algorithm was implemented and the fault coverage was observed to be the same as SS. A reduction in test vectors up to $60 \%$ can be observed (Table 2) in most of the circuits. Maximum reduction is acheived when the average number of faults per combinational vector is small and the number of FFs is proportionally higher. Since in these cases the setup time of scan FFs would increase compared to RAS. The reduction in test time is slightly lower than that described in [18]. This is because of the improvement that we made in the design, by minimizing the number of signals that needs to be routed to every FF.

Relative reduction of power dissipation in the circuit is calculated assuming that, the power dissipated is directly proportional to the number of transitions in the primary inputs and states of FFs. The results were obtained for both SS and RAS (Table 3). It can be observed that, as the size of the circuits increases, reduction in power dissipation up to $99 \%$ is achieved using RAS.

\section{Modifying ATPG to Decrease Num- ber of Vectors}

The results presented in this paper are based on the vectors obtained using existing ATPG algoritms. A slight modification in the form of an added constrain in the ATPG, can further decrease the test vectors using RAS. The following algorithm can be employed to obtain further compaction of test vectors. 
Table 2. Results of Vector Compaction for various Benchmark Circuits.

\begin{tabular}{|c||c|c|c|c|c|}
\hline Circuit & $\begin{array}{c}\text { No. } \\
\text { of } \\
\text { FFs }\end{array}$ & $\begin{array}{c}\text { No. of } \\
\text { Combi. } \\
\text { vectors }\end{array}$ & $\begin{array}{c}\text { No. of } \\
\text { clock cycles } \\
\text { in SS }\end{array}$ & $\begin{array}{c}\text { No. of } \\
\text { clock cycles } \\
\text { in RAS }\end{array}$ & $\begin{array}{c}\text { Test } \\
\text { time } \\
\text { red. (\%) }\end{array}$ \\
\hline \hline s208 & 8 & 64 & 584 & 301 & 48.46 \\
\hline s349 & 11 & 42 & 687 & 366 & 46.72 \\
\hline s386 & 6 & 138 & 972 & 450 & 53.70 \\
\hline s420 & 16 & 128 & 2192 & 1056 & 51.82 \\
\hline s510 & 6 & 110 & 776 & 344 & 55.67 \\
\hline s641 & 19 & 142 & 2859 & 1148 & 59.85 \\
\hline s838 & 32 & 240 & 7952 & 3595 & 54.79 \\
\hline s1196 & 18 & 344 & 6554 & 2447 & 62.66 \\
\hline s1269 & 37 & 118 & 4521 & 1981 & 56.18 \\
\hline s3271 & 116 & 264 & 31004 & 12540 & 59.55 \\
\hline s3384 & 183 & 260 & 48759 & 21119 & 56.69 \\
\hline s5378 & 179 & 618 & 111419 & 48677 & 56.31 \\
\hline s13207 & 638 & 1138 & 727820 & 309132 & 57.53 \\
\hline
\end{tabular}

1. Set the cost function of setting a FF to be highest

2. Generate a vector to target a fault

3. Perform Fault simulation

Table 3. Power estimation based on number

4. While number of faults $>0$ of transitions at the inputs for various Benchmark Circuits.

\begin{tabular}{|c||c|c|c|}
\hline Circuit & $\begin{array}{c}\text { No. of } \\
\text { Tansitions } \\
\text { in SS tests }\end{array}$ & $\begin{array}{c}\text { No. of } \\
\text { Transitions } \\
\text { in RAS tests }\end{array}$ & $\begin{array}{c}\text { Test } \\
\text { power } \\
\text { saving (\%) }\end{array}$ \\
\hline \hline s208 & 1866 & 1209 & 35.21 \\
\hline s349 & 4755 & 1233 & 74.07 \\
\hline s386 & 2495 & 1515 & 39.28 \\
\hline s420 & 11587 & 4708 & 59.37 \\
\hline s510 & 3141 & 2382 & 24.16 \\
\hline s641 & 27715 & 7924 & 71.41 \\
\hline s838 & 72914 & 17782 & 75.61 \\
\hline s1196 & 57409 & 10601 & 81.53 \\
\hline s1269 & 77755 & 7880 & 89.87 \\
\hline s3271 & 1744149 & 45971 & 97.36 \\
\hline s3384 & 4299362 & 77665 & 98.19 \\
\hline s5378 & 8947677 & 175710 & 98.04 \\
\hline s13207 & 230176409 & 211048 & 99.91 \\
\hline
\end{tabular}

(a) Read all the FF where the faults are detected

(b) Target a fault and Generate the next vector with minimum changes to be made in the FFs from the current states.

(c) Perform Fault simulation

\section{End While}

Let us consider modifying PODEM's [30] backtrace algorithm, where the pseudo-controllability ${ }^{2}$ of the FF (pseudo primary inputs) is set very high. Hence during backtrace, minimal set of FFs are set for a targeted fault. This algorithm was implemented on s27 circuit, in which 12 vectors were generated by the combinational ATPG, and applying the constraints of the algorithm resulted in 16 vectors which is a reduction of $43 \%$ compared to the results obtained when a vector reordering scheme was used earlier and $68 \%$ decrease from the number of scan vectors. Its worthwhile noting that, better vector compaction can be acheived for larger circuits using this algorithm.

\footnotetext{
${ }^{2} \mathrm{FFs}$ are actually controllable here, but a minimum set of FF changes is targeted
} 


\section{Conclusion}

RAS has started gaining acceptance gradually. A practically implementable architecture for RAS is proposed here. An algorithm is constructed to re-order and compact the test vectors. The flexibility of the design helps to detect non-targeted faults, since any arbitrary vector can be applied and any arbitrary FF can be observed. Simulation results show that power dissipation is reduced up to $99 \%$, and up to $60 \%$ reduction in test vectors is observed compared to the Serial Scan. Test application time as well as power consumption in a circuit are complementary objectives in $\mathrm{SS}$, but are addressed concurrently in RAS, where both are reduced simultaneously. This work is based on the premise that as technology improves and test complexity increases, a marginal increase in chip area for design for testability is least prohibitive.

\section{References}

[1] V. D. Agrawal, K.-T. Cheng, D. D. Johnson, and T. Lin, "Designing Circuits with Partial Scan," IEEE Design \& Test of Computers, vol. 5, pp. 815, Apr. 1988.

[2] S. J. Chandra, T. Ferry, T. Gheewala, and K. Pierce, "ATPG Based on a Novel Grid Addressable Latch Element," in Proc. ACM/IEEE Design Automation Conf., pp. 282-286.

[3] R. M. Chou, K. K. Saluja, and V. D. Agrawal, "Power Constraint scheduling of tests," in Proc. 7th International Conference VLSI Design, pp. 271-274, Jan. 1994.

[4] R. M. Chou, K. K. Saluja, and V. D. Agrawal, "Scheduling Tests for VLSI Systems Under Power Constraints," IEEE Trans. VLSI Systems, vol. 5, pp. 175-185, June 1997.

[5] A. Chandra and K. Chakrabarty, "Combining Low-Power Scan Testing and Test Data Compression for System-on-a-chip," in Proc. Design Automation Conf., pp. 166-169.

[6] S. Wang and S. K. Gupta, "ATPG For Heat Dissipation Minimization During Scan Testing," in Proc. Design Automation Conf., pp. 614-619.
[7] R. Sankaralingam, R. R. Oruganti, and N. A. Touba, "Static Compaction Techniques to Control Scan Vector Power Dissipation," in Proc. VLSI Test Symposium, pp. 35-40.

[8] S. Kajihara, K. Ishida, and K. Miyase, "Test Vector Modification for Power Reduction During Scan Testing," in Proc. in VLSI Test Symposium, pp. 160-165.

[9] V. Dabholkar, S. Chakravarty, I. Pomeranz, and S. M. Reddy, "Techniques for Minimizing Power Dissipation in Scan and Combinational Circuits during Test Application," in IEEE Tran. on Computer-Aided Design of Integrated Circuits and Systems, pp. 1325-1333.

[10] B. Bhattacharya, S. Seth, and S. Zhang, "DoubleTree scan: a novel low-power scan-path architecture," in Proc. International Test Conference, pp. $470-479$.

[11] I. Hamzaoglu and J. Patel, "Reducing Test Application Time for Full Scan Embedded Cores," in Proc. FTCS, pp. 260-267.

[12] M. L. Bushnell and V. D. Agrawal, Essentials of Electronic Testing for Digital, Memory and Mixed-Signal VLSI Circuits. Boston: Kluwer Academic Publishers, 2000.

[13] K.-T. Cheng, S. Devadas, and K. Keutzer, "Delay Fault Test Generation and Synthesis for Testability Under a Standard Scan Design Methodology," IEEE Trans. on Computer-Aided Design, vol. 12, pp. 1217-1231, Aug. 1993.

[14] J. Savir, "Skewed-Load Transition Test: Part I, Calculus," in Proc. International Test Conf., pp. 705-713.

[15] J. Savir, "Skewed-Load Transition Test: Part II, Coverage," in Proc. International Test Conf., pp. $714-722$.

[16] J. Savir, "On Broad-Side Delay Testing," in Proc. 12th VLSI Test Symp., pp. 284-290.

[17] H. Ando, "Testing VLSI with Random Access Scan," in Proc. COMPCON, pp. 50-52, Feb. 1980.

[18] D. H. Baik, K. K. Saluja, and S. Kajihara, "Random Access Scan: A Solution to Test Power, Test Data Volume and Test Time," in Proc. 17th International Conf. VLSI Design, pp. 883-888, Jan. 2004. 
[19] B. Arslan and A. Orailoglu, "Test Cost Reduction through a Reconfigurable Scan Architecture," in Proc. International Test Conference, pp. 945-952, Oct. 2004.

[20] Z. Plíva, O. Novák, and P. B. d'Aguerre, "Hardware overhead of Boundry Scan and RAS Design Methodologies.".

[21] T. J. Powell, "Consistently Dominant Fault Model for Tristate Buffer Nets," in Proc. VLSI Test Symp., pp. 400-404.

[22] S. T. Chakradhar, S. G. Rothweiler, and V. D. Agrawal, "Redundancy Removal and Test Generation for Circuits with Non-Boolean Primitives," IEEE Trans. CAD, vol. 16, pp. 1370-1377, Nov. 1997.

[23] A. J. van de Goor, Testing Semiconductor Memories: Theory and Practice. Chichester, UK: John Wiley \& Sons, Inc., 1991.

[24] T. M. Niermann and J. H. Patel, "HITEC: A Test Generation Package for Sequential Circuits," in Proc. European Design Automation Conference, pp. 214-218.
[25] C. E. Stroud, "AUSIM: Auburn University SIMulator - Version L2.2." Dept. of Electrical \& Computer Engineering, Auburn University, Jan. 2004.

[26] S. Gerstendorfer and H. J. Wunderlich, "Minimised Power Consumption for Scan-based BIST," in Proc. International Test Conference, pp. 77-84.

[27] X. Zhang and K. Roy, "Power Reduction in TestPer-Scan BIST," in Proc. International On Line Testing Workshop, pp. 133-138.

[28] S. DasGupta, R. G. Walther, and T. W. Williams, "An Enhancement to LSSD and Some Applications of LSSD in Reliability," in Proc. International Fault-Tolerant Computing Symp., pp. 3234 .

[29] M. A. Gharaybeh, M. L. Bushnell, and V. D. Agrawal, "Classification and Test Generation for Path-Delay Faults Using Single Stuck-at Fault Tests," J. Electronic Testing: Theory and Applications, vol. 11, pp. 55-67, Aug. 1997.

[30] P. Goel, "An Implicit Enumeration Algorithm to Generate Tests for Combinational Logic Circuits," IEEE Trans. on Computers, vol. C-30, pp. 215222, Mar. 1981. 